M. OZAWA

KODAI MATH. J.

3 (1980), 26-39

\title{
HILBERT $B(H)$-MODULES AND STATIONARY PROCESSES
}

\author{
By Masanao Ozawa
}

\section{Introduction.}

In the theory of multivariate or Hilbert space valued stationary processes, the Gram matricial structure of the time domain of processes plays an important role. The Gram matricial structure of $q$-variate processes forms a module over a ring of all $q \times q$-matrices with a $q \times q$-matrix valued inner product, which can be seen as a Hilbert space over a matrix ring but not over the complex number field (cf. Masani [5]). Thus it is desirable to formulate such a structure abstractly free from the underlying probabilistic structure, as Kolmogorov first emphasized in 1940 for the univariate case where unspecified Hilbert spaces are prefered to $L^{2}$-spaces (cf. [4]). The purpose of the present paper is to establish such an abstact concept of the time domain of processes.

In the next section, we shall give definitions of Hilbert $B(H)$-modules and stationary processes on them. Our definition of a Hilbert $B(H)$-module is similar to the Paschke's definition [6] of inner product modules over $B^{*}$ algebras except that we require the range of the inner product, which we call the Gramian, is contained in the trace class. Such a requirement is always satisfied for the setting of $q$-variate or Hilbert space valued processes, and plays an essential role in our treatment. In Sect. 3 we shall study, in a general setting, positive sesquilinear maps valued in the predual of a $W^{*}$ algebra, example of which are the Gramian and the operator valued covariance function of stationary processes, and we shall examine the relation with the *-representation and construct a unitary representation which is a module version of Umegaki's construction [10], which are applied to the later sections. In Sect. 4 we shall show that the structure of Hilbert $B(H)$-modules is completely determined by the power of their modular bases, and that a Fourier expansion by the modular basis and Gramian is possible in a parallel way with one on the usual Hilbert spaces. In Sect. 5 applying a general theorem obtained in Sect. 3, the equivalence of stationary processes on Hilbert $B(H)$-modules is established by their covariance functions.

The author would like to express his thanks to Professor H. Umegaki for his valuable advices and encouragements.

Received October 25, 1978 


\section{Hilbert $B(H)$-modules and $G$-stationary processes.}

Let $H$ be a Hilbert space over a complex number field $C$, let $B(H)$ be the algebra of all bounded operators on $H$, and let $T(H)$ be the ideal of $B(H)$ consisting of all trace class operators on $H$. For a left $B(H)$-module $X$ we will denote the action of $B(H)$ on $X$ by $a, x \rightarrow a \cdot x$.

Definition 2.1. A pre-Hilbert $B(H)$-module is a left $B(H)$-module $X$ equipped with a map $[\cdot, \cdot] ; X \times X \rightarrow T(H)$ satisfying:

(i) $[x+y, z]=[x, z]+[y, z]$,

(ii) $[a \cdot x, y]=a[x, y]$,

(iii) $[x, y]^{*}=[y, x]$,

(iv) $[x, x] \geqq 0$, and $[x, x]=0$ only if $x=0$,

for all $x, y, z$ in $X, a$ in $B(H)$. The map $[\cdot, \cdot]$ will be called the Gramian on $X$.

It is easy to see that $1 \cdot x=x$ for the identity 1 in $B(H), x$ in $X$, and that $[x, a \cdot y]=[x, y] a^{*}$ for all $a$ in $B(H), x, y$ in $X$ (cf. [6]).

For a pre-Hilbert $B(H)$-module $X$, we define the scalar multiplication by $\alpha x=(\alpha 1) \cdot x$ for complex $\alpha, x$ in $X$, the inner product by $(x, y)=\operatorname{Tr}[x, y]$, and the norm by $\|x\|_{2}=(x, x)^{1 / 2}$. Then $X$ has also a pre-Hilbert space structure. A pre-Hilbert $B(H)$-module which is complete with respect to the norm $\|\cdot\|_{2}$ is called a Hilbert $B(H)$-module.

When $H$ is one-dimensional, we have $B(H)=T(H)=C$, and hence the concept of a Hilbert $B(H)$-module coincides with that of a Hilbert space.

The Hilbert space $H$ itself is a simple but important example of a Hilbert $B(H)$-module. In fact, for each $\xi, \eta$ in $H$, denote by $\xi \otimes \vec{\eta}$ the operator on $H$ given by

$$
(\xi \otimes \bar{\eta}) \zeta=(\zeta, \eta) \xi
$$

for all $\zeta$ in $H$, as in $[9]$, then $[\xi, \eta]=\xi \otimes \bar{\eta}$ defines a Gramian on $H$, under the natural action of $B(H)$ on $H$, and the inner product given by $\operatorname{Tr}[x, y]$ coincides with the original one. Moreover, we can show that every Gramian on the left $B(H)$-module $H$ is of this form if $\operatorname{Tr}[\xi, \eta]=(\xi, \eta)$, as follows.

Proposition 2.2. Let $H$ be a left $B(H)$-module with natural action, and $F$ be a $T(H)$-valued function satisfying the defining conditions of the Gramian on $H$. Then there is a positve $\lambda$ such that $F[\xi, \eta]=\lambda \xi \otimes \bar{\eta}$ for all $\xi, \eta$ in $H$. If $\operatorname{Tr} F[\xi, \xi]=(\xi, \xi)$ for some $\xi$ then $\lambda=1$.

Proof. Let $\phi$ be a unit vector in $H$. Then we have that $F[\phi, \phi] \geqq 0,=0$ and that

$$
F[\phi, \phi]=F[(\phi \otimes \bar{\phi}) \phi,(\phi \otimes \bar{\phi}) \phi]=\phi \otimes \bar{\phi} F[\phi, \phi] \phi \otimes \bar{\phi},
$$

and hence there is a positive $\lambda$ such that $F[\phi, \phi]=\lambda \phi \otimes \bar{\phi}$. For any $\xi, \gamma$ in $H$, we have that

$F[\xi, \eta]=F[(\xi \otimes \bar{\phi}) \phi,(\eta \otimes \bar{\phi}) \phi]=\xi \otimes \bar{\phi} F[\phi, \phi] \phi \otimes \bar{\eta}=\lambda \xi \otimes \bar{\eta}$, 
and that $\operatorname{Tr} F[\xi, \eta]=\lambda(\xi, \eta)$.

Q.E.D.

Definition 2.3. Let $X$ be a Hilbert $B(H)$-module, and let $G$ be a locally compact group. A family $\left\{x_{t} ; t\right.$ in $\left.G\right\}$ of elements of $X$ is called a $G$-stationary process on $X$ if the following conditions are satisfied

(i) the Gramian $\left[x_{s}, x_{t}\right]$ depends only on $t^{-1} s$,

(ii) the function $t \rightarrow\left[x_{t}, x_{e}\right]$ is weakly continuous,

(iii) $\left\{x_{t} ; t\right.$ in $\left.G\right\}$ spans $X$, that is, the smallest closed submodule containing $\left\{x_{t} ; t\right.$ in $\left.G\right\}$ is $X$. The function $\Gamma(t)=\left[x_{t}, x_{e}\right]$ is called the covariance function of $\left\{x_{t}\right\}$.

Definition 2.4. Let $X$ and $Y$ be two Hilbert $B(H)$-modules with Gramians $[\cdot, \cdot]_{X}$ and $[\cdot, \cdot]_{Y}$, respectively. A map $U$ from $X$ onto $Y$ is called an $\imath$ somorphısm if $U$ satisfies that

(i) $U(x+y)=U x+U y$,

(ii) $U(a \cdot x)=a \cdot U x$,

(iii) $[U x, U y]_{Y}=[x, y]_{X}$,

for all $x, y$ in $X, a$ in $B(H)$. We say that two Hilbert $B(H)$-modules are equivalent if there is an isomorphism from one onto another. Let $\left\{x_{t}\right\}$ and $\left\{y_{t}\right\}$ be two $G$-stationary processes on $X$ and $Y$, respectively. We say that $\left\{x_{t}\right\}$ and $\left\{y_{t}\right\}$ are equivalent if there is an isomorphism $U$ from $X$ onto $Y$ such that $U x_{t}=y_{t}$, for all $t$ in $G$.

Our formulations of Hilbert $B(H)$-modules and $G$-stationary processes may provide a nice setting for the Hilbert space valued stationary processes, in view of the following examples.

EXAMPLE 2.5. Let $K^{q}$ be the Cartesian product of a Hilbert space $K$ with itself $n$ times, i.e., the set of all vectors $x=\left(x_{1}, \cdots, x_{q}\right)$ such that each $x_{2}$ is in $K$. For $x, y$ in $K^{q}$, the $q \times q$-matrix $\left(a_{\imath j}\right)$ defined by $a_{\imath j}=\left(x_{\imath}, y_{j}\right)$ is called the Gramian of the ordered pair $x, y$. Then $K^{q}$ is a Hilbert $B\left(C^{q}\right)$-module with Gramian $[x, y]=\left(a_{\imath j}\right)$, as explained in the Masani's survey [p. $\left.353 ; 5\right]$.

EXAMPLE 2.6. Let $(\Omega, P)$ be a probability measure space, let $H$ be a separable Hilbert space, and let $L^{2}(H)$ the Hilbert space of all square Bochner integrable $H$-valued functions on $(\Omega, P)$. Then it is easy to see that $L^{2}(H)$ is a left $B(H)$-module in the obvious way. For any pair $x, y$ in $L^{2}(H)$ there corresponds a unique trace class operator $[x, y]$ such that

$$
([x, y] \xi, \eta)=\int_{\Omega}(\xi, y(\omega))(x(\omega), \eta) P(d \omega),
$$

and that $\operatorname{Tr}[x, y]=\int_{\Omega}(x(\omega), y(\omega)) P(d \omega)$. Then it is easy to see that $L^{2}(H)$ is a Hilbert $B(H)$-module with Gramian $[x, y]$, whose properties are investigated 
in Umegaki [11] in connection with the tensor product Hilbert space. Let $\left\{x_{t} ; t\right.$ in $\left.R\right\}$ be a family of $H$-valued random variables in $L^{2}(H)$ such that $\left[x_{s}, x_{t}\right]$ depens only on $s-t$, and $\Gamma(t)=\left[x_{t}, x_{0}\right]$ is weakly continuous. Then $\left\{x_{t}\right\}$ is called the $H$-valued stationary process. In this case usually the time domain $X$ of the process $\left\{x_{t}\right\}$ is defined as the closed submodule of $L^{2}(H)$ spanned by $\left\{x_{t}\right\}$. Thus the $H$-valued stationary process $\left\{x_{t}\right\}$ with time domain $X$ is a $R$-stationary process on $X$ in our sense. Further information on such a process will be found in many literatures, for instance [3].

EXAMPLE 2.7. Let $S(K, H)$ be the set of all bounded linear transformations $x$ from a Hilbert space $K$ to a Hilbert space $H$, such that $x x^{*}$ is a trace class operator on $H$ where $x^{*}$ is a bounded linear transformation from $H$ to $K$ defined by the relation $(x * \xi, \eta)=(\xi, x \eta)$ for all $\xi$ in $H, \eta$ in $K$. Then it is easy to see that $S(K, H)$ is a Hilbert $B(H)$-module with Gramian $[x, y]=x y^{*}$. We call this the Hilbert $B(H)$-module $S(K, H)$.

\section{Positive sesquilinear maps.}

In order to provide some technical results used in the later sections, we shall study positive sesquilinear maps with values in the predual of a $W^{*}$-algebra in this section.

Let $P$ be a linear map from a $C^{*}$-algebra $A$ into a $C^{*}$-algebra $B$. Let $M_{n}(A)$ be the $C^{*}$-algebra of all $n \times n$-matrices with entries in $A$, and let $P_{n}$ be the linear map from $M_{n}(A)$ into $M_{n}(B)$ obtained by applying $P$ to each entry of an element of $M_{n}(A)$. We say that $P$ is $n$-positive if $P_{n}$ maps positive elements in $M_{n}(A)$ into positive elements in $M_{n}(B)$, and that $P$ is completely positive if $P$ is $n$-positive for each positive integer $n$. It should be remarked [6] that $P$ in $n$-positive if and only if $\sum_{i, j} b_{i} * P\left(a_{\imath} * a_{j}\right) b_{j} \geqq 0$ for all $a_{1}, \cdots, a_{n}$ in $A, b_{1}, \cdots, b_{n}$ in $B$.

Let $M$ be a $W^{*}$-algebra, that is, $M$ is a $C^{*}$-algebra which is a dual space of a Banach space $M_{*}$. We denote the norm on $M$ and $M_{*}$ by $\|\cdot\|_{\infty}$ and $\|\cdot\|_{1}$ respectively, and by $\langle\cdot, \cdot\rangle$ the dual pair on $M \times M_{*}$. For $f$ in $M_{*}$ and $a$ in $M$, we denote by $f^{*}, a \cdot f$ and $f \cdot a$, the elements of $M_{*}$ defined by the relations $\left\langle b, f^{*}\right\rangle=\overline{\left\langle b^{*}, f\right\rangle},\langle b, a \cdot f\rangle=\langle b a, f\rangle$ and $\langle b, f \cdot a\rangle=\langle a b, f\rangle$ for all $b$ in $M$. For the case in which $M=B(H), M_{*}$ can be regarded as $T(H)$. In this case, $\langle a, f\rangle$ $=\operatorname{Tr} a f, f^{*}$ is the adjoint of $f, a \cdot f=a f$ and $f \cdot a=f a$ for all $a$ in $B(H)$ and $f$ in $T(H)$.

Let $L$ be a linear space. We say that $F$ is an $M_{*}$-sesquilinear form on $L$

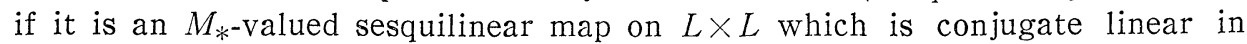
the second variable. In the following we consider an $M_{*}$-sesquilinear form $F$ on $L$, and write $[x, y]$ for $F(x, y)$ if no confusion may occur. For a positive integer $n, F$ is said to be $n$-positvve (or positıve, when $n=1$ ) if

$$
\sum_{\imath, j}\left\langle a_{\jmath}^{*} a_{\imath},\left[x_{\imath}, x_{\jmath}\right]\right\rangle \geqq 0
$$


for all $a_{1}, \cdots, a_{n}$ in $M, x_{1}, \cdots, x_{n}$ in $L . F$ is said to be completely positive if $F$ is $n$-positive for all positive integer $n$. It is easy to see that $F$ is $n$-positive if and only if $\sum_{\imath, j} a_{\imath} \cdot\left[x_{\imath}, x_{\jmath}\right] \cdot a_{\jmath}{ }^{*} \geqq 0$ for all $a_{1}, \cdots, a_{n}$ in $M, x_{1}, \cdots, x_{n}$ in $L$.

LEMMA 3.1. Let $N$ be a self-adjoint subalgebra of a $W^{*}$-algebra $M$ which is $\sigma\left(M, M_{*}\right)$-dense in $M$. Then an $M_{*}$-sesquilinear form $F$ on $L$ is n-positvve if it satisfies the inequality (1) for all $a_{1}, \cdots, a_{n}$ in $N, x_{1}, \cdots, x_{n}$ in $L$.

Proof. Let $S$ be a unit sphere of $M$. Then by Kaplansky's density theorem, $N \cap S$ is $s\left(M, M_{*}\right)$-dense in $S$. Since the multiplication on $M$ is jointly $s\left(M, M_{*}\right)$ continuous on $S$, it is easy to see that the inequality (1) holds for all $a_{1}, \cdots$, $a_{n}$ in $S$, and hence multiplying positive numbers it holds for all $a_{1}, \cdots, a_{n}$ in $M$.

Q.E. D

Obviously $n$-positivity follows from $n+1$-positivity, and every positive $M_{*}$-sesquilinear form is symmetric in the sense that $[x, y]=[y, x]^{*}$ for all $x, y$ in $L$. For a positive $M_{*}$-sesquilinear form $F$, each $a$ in $M$ defines a semidefinite inner product $x, y \rightarrow\left\langle a^{*} a,[x, y]\right\rangle$ on $L$, and so by the usual Schwartz inequality we have that

$$
\left|\left\langle a^{*} a,[x, y]\right\rangle\right|^{2} \leqq\left\langle a^{*} a,[x, x]\right\rangle\left\langle a^{*} a,[y, y]\right\rangle
$$

for all $x, y$ in $L$. But a more delicate form of a Schwartz inequality characterizes the 2-positivity of $F$.

THeorem. 3.2. Let $F$ be a positive $M_{*}$-sesquilinear form on $L$. Then $F$ is 2-positive if and only if

$$
\left|\left\langle b^{*} a,[x, y]\right\rangle\right|^{2} \leqq\left\langle a^{*} a,[x, x]\right\rangle\left\langle b^{*} b,[y, y]\right\rangle
$$

for all $a, b$ in $M, x, y$ in $L$.

Proof. Suppose that $F$ is 2-positive. Let $t$ be a real number, and $\alpha$ be such that $\alpha=t\left|\left\langle b^{*} a,[x, y]\right\rangle\right|\left\langle b^{*} a,[x, y]\right\rangle^{-1}$. Then we have for $a_{1}=a, a_{2}=b$, $x_{1}=\alpha x, x_{2}=y$,

$$
\begin{aligned}
0 & \leqq \sum_{\imath, j=1}^{2}\left\langle a_{\jmath} a_{\imath},\left[x_{\imath}, x_{\jmath}\right]\right\rangle \\
& =t^{2}\left\langle a^{*} a,[x, x]\right\rangle+2 t\left|\left\langle b^{*} a,[x, y]\right\rangle\right|+\left\langle b^{*} b,[y, y]\right\rangle .
\end{aligned}
$$

Since $t$ is arbitrary, we have the required inequality. Conversely, if the inequality (2) holds, we have

$$
\begin{aligned}
\sum_{\imath, j=1}^{2}\left\langle a_{\jmath} *\right. & \left.a_{\imath},\left[x_{\imath}, x_{\jmath}\right]\right\rangle \\
& =\sum_{\imath=1}^{2}\left\langle a_{\imath}{ }^{*} a_{\imath},\left[x_{\imath}, x_{\imath}\right]\right\rangle+2 \operatorname{Re}\left\langle a_{2}{ }^{*} a_{1},\left[x_{1}, x_{2}\right]\right\rangle \\
& \geqq 2\left(\left\langle a_{1} * a_{1},\left[x_{1}, x_{1}\right]\right\rangle\left\langle a_{2}{ }^{*} a_{2},\left[x_{2}, x_{2}\right]\right\rangle\right)^{1 / 2}+2 \operatorname{Re}\left\langle a_{2}{ }^{*} a_{1},\left[x_{1}, x_{2}\right]\right\rangle \\
& \geqq 2\left(\left|\left\langle a_{2}{ }^{*} a_{1},\left[x_{1}, x_{2}\right]\right\rangle\right|+\operatorname{Re}\left\langle a_{2}^{*} a_{1},\left[x_{1}, x_{2}\right]\right\rangle\right) \geqq 0 . \quad \text { Q. E. D. }
\end{aligned}
$$


From the above inequality we can constitute an elementary proof of M. D. Choi's inequality $P\left(a^{*} a\right) \geqq P\left(a^{*}\right) P(a)$ for 2-positive unit-preserving linear maps on a $C^{*}$-algebra.

Corollary 3.3. If $P$ is a 2-positive linear map from a $C^{*}$-algebra $A$ into a $C^{*}$-algebra $B$, then $\|P\| P\left(a^{*} a\right) \geqq P\left(a^{*}\right) P(a)$ for all $a$ in $A$, and $\|P\|=\|P(1)\|$ if $A$ is unital.

Proof. Without any loss of generality, we assume $B$ is faithfully represented on a Hilbert space $L$, and that $P$ is a restriction of a normal linear map $P^{\prime}$ on $A^{* *}$ into $B(L)$. Define $A^{*}$-sesquilinear form $[\cdot, \cdot]$ on $L$ by the relation $\langle a,[x, y]\rangle=\left(P^{\prime}(a) x, y\right)$ for all $a$ in $A^{* *}, x, y$ in $L$. Then it is easy to see that $[\cdot, \cdot]$ is a 2 -positive $A^{*}$-sesquilinear form, from the 2-positivity of $P$ and Lemma 3.1. Thus applying the inequality (2) for $b=1$ in $A^{* *}, a$ in $A$, $y=P(a) x$, the routine calculus shows that $\|P(a) x\|^{2} \leqq\left\|P^{\prime}(1)\right\|\left(P\left(a^{*} a\right) x, x\right)$, so that $\left\|P^{\prime}(1)\right\| P\left(a^{*} a\right) \geqq P\left(a^{*}\right) P(a)$. It follows that $\|P(a)\|^{2}=\left\|P\left(a^{*}\right) P(a)\right\| \leqq$ $\left\|P^{\prime}(1)\right\|\left\|P\left(a^{*} a\right)\right\| \leqq\left\|P^{\prime}(1)\right\|\|P\|\|a\|^{2}$, and thus $\|P\|=\left\|P^{\prime}(1)\right\| . \quad$ Q. E. D.

In the following, we denote by $(\cdot, \cdot)$ the semidefinite inner product on $L$ given by $(x, y)=\langle 1,[x, y]\rangle$ and by $\|\cdot\|_{2}$ the seminorm on $L$ given by $\|x\|_{2}^{2}=\|[x, x]\|_{1}$.

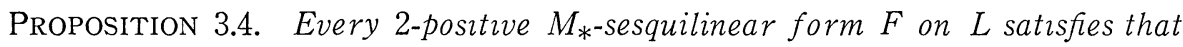

for all $x, y$ in $L$.

$$
\|[x, y]\|_{1}=\|x\|_{2}\|y\|_{2}
$$

Proof. By the polar decomposition of elements of $M_{*}[1.14 .4 ; 8]$ there is a partial isometry $u$ in $M$ such that $\|[x, y]\|_{1}=\langle u,[x, y]\rangle$. Thus a simple application of the inequality (2) concludes the inequality (3). Q. E.D.

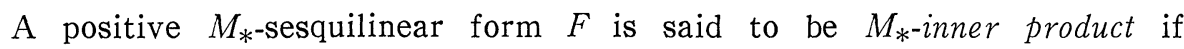
$[x, x]=0$ implies $x=0$. From every positive $M_{*}$-sesquilinear form $F$ on $L$, we can obtain an $M_{*}$-inner product on the factor space $L / N$ where $N=\{x$ in $L$; $[x, x]=0\}$ as $[x+N, y+N]=[x, y]$. If $F$ is a 2-positive $M_{*}$-inner product on $L$, then the completion $\bar{L}$ of $L$ by the norm $\|\cdot\|_{2}$ is a Hilbert space, to which we can extend $F$ uniquely by continuity shown in Proposition 3.4.

THEOREM 3.5. Let $F$ be an $n$-positive ( $n \geqq 2) M_{*}$-sesquilinear form on $L$, and $W$ be the Hilbert space obtained by factoring and completing $L$. Then there is a uuique untt-preserving normal n-positvve linear map $\rho$ on $M$ into $B(W)$ such that

$$
(\rho(a) \dot{x}, \dot{y})=\langle a,[x, y]\rangle
$$

for all a in $M, x, y$ in $L$, where $\dot{x}, \dot{y}$ are the corresponding elements in the factor space, which is a ${ }^{*}$-representation if and only if

$$
[\rho(a) \dot{x}, \dot{y}]=a \cdot[x, y]
$$

for all $a$ in $M$ and $x$ in $L$. 
Proof. For each $a$ in $M$, by the inequality (2) we have

$$
|\langle a,[x, y]\rangle| \leqq\|a\|_{\infty}\|x\|_{2}\|y\|_{2}
$$

for all $x, y$ in $L$. Thus the sesquilinear form $\dot{x}, \dot{y} \rightarrow\langle a,[x, y]\rangle$ on the factor space defines a unique bounded linear operator $\rho(a)$ on $W$ such that $(\rho(a) \dot{x}, \dot{y})$ $=\langle a,[x, y]\rangle$ for all $x, y$ in $L$. Now to check the required properties of the map $\rho: a \rightarrow \rho(a)$ is a matter of routine calculation. The last part of the assertion follows from the following relations using the fact that $[x, y]=[\dot{x}, \dot{y}]$,

$$
(\rho(a b) \dot{x}, \dot{y})=\langle a b,[x, y]\rangle,\langle a,[\rho(b) \dot{x}, \dot{y}]\rangle=(\rho(a) \rho(b) \dot{x}, \dot{y})
$$

for all $a, b$ in $M, x, y$ in $L$. Thus the proof is completed.

Q.E.D.

The map $\rho$ will be called the assoicrate map of $F$.

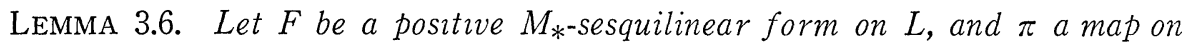
$M$ whose values are linear transformations on $L$ such that $[\pi(a) x, y]=a \cdot[x, y]$ for all $a$ in $M, x, y$ in $L$. Then $\pi$ induces a non-degenerate normal *-representation of $M$ on the Hilbert space $W$ obtained by factoring and completing $L$.

Proof. First we observe that $[\pi(a) x, \pi(b) y]=a \cdot[x, y] \cdot b^{*}$. It follows that $F$ is completely positive and that the set $N=\{x$ in $L:[x, x]=0\}$ is invariant under $\pi(a)$. Thus we can consider that $\pi(a)$ acts on the factor space $L / N$. If $\rho$ is the associate map of $F$, then we have that

$$
(\rho(a) \dot{x}, \dot{y})=\langle a,[x, y]\rangle=\langle 1, a \cdot[x, y]\rangle=(\pi(a) \dot{x}, \dot{y})
$$

for all $x, y$ in $L$. Thus $\rho=\pi$, and the conclusion follows from Theorem 3.5.

Q.E.D.

Let $F$ be a completely positive $M_{*}$-sesquilinear form on $L$, and $M \otimes L$ be

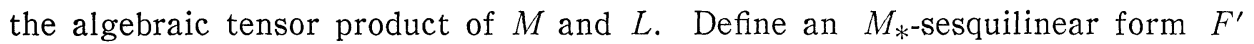
on $M \otimes L$ by

$$
F^{\prime}\left[\sum_{\imath} a_{i} \otimes x_{\imath}, \sum_{j} b_{j} \otimes y_{j}\right]=\sum_{i, j} a_{\imath} \cdot F\left[x_{\imath}, y_{j}\right] \cdot b_{j}{ }^{*}
$$

Then by the complete positively of $F, F^{\prime}$ is positive. Let $\pi(a)$ be the linear map on $M \otimes L$ such that

$$
\pi(a) \sum_{\boldsymbol{i}} a_{i} \otimes x_{\imath}=\sum_{\boldsymbol{i}} a a_{\imath} \otimes x_{\imath}
$$

Then clearly $F^{\prime}[\pi(a) x, y]=a \cdot F^{\prime}[x, y]$ for all $x, y$ in $M \otimes L$, and hence by Lemma 3.6, $\pi$ induces a non-degenerate normal *-representation on the Hilbert space obtained by factoring and completing $M \otimes L$. This construction of a *-representation is essentially same to that found by Stinespring [12].

Let $G$ be a locally compact group. An $M_{*}$-valued function $V$ on $G$ is said to be positive definite if it satisfies that

$$
\sum_{i, j}\left\langle a_{j}^{*} a_{\imath}, V\left(t_{j}^{-1} t_{\imath}\right)\right\rangle \geqq 0
$$


for all positive integer $n, a_{1}, \cdots, a_{n}$ in $M$, and $t_{1}, \cdots, t_{n}$ in $G$, and it is said to be weakly continuous if $t \rightarrow\langle a, V(t)\rangle$ is continuous on $G$ for all $a$ in $M$.

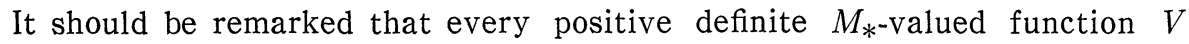
can be extended to the completely positive $M_{*}$-sesquilinear form $F$ on the linear space $F(G)$ of all complex-valued functions on $G$ with finite support, which satisfies that

$$
F[x, y]=\sum_{s} \sum_{t} x(s) \overline{y(t)} V\left(t^{-1} s\right)
$$

for all $x, y$ in $F(G)$.

THEOREM 3.7. Let $V$ be a weakly contrnuous positive definte $M_{*}$-valued function on $G$. Then there is a Hilbert space $W$, a non-degenerate normal *representation $\pi$ of $M$ on $W$, a strongly continuous unitary representation $U$ of $G$ on $W$ and a vector $\xi$ in $W$ satisfying.

(i) $U(t)$ is in $\pi(M)^{\prime}$ for all $t$ in $G$,

(ii) the linear span of the set $\{\pi(a) U(t) \xi ; a$ in $M, t$ in $G\}$ is dense in $W$,

(iii) $\langle a, V(t)\rangle=(\pi(a) U(t) \xi, \xi)$ for all $a$ in $M$ and $t$ in $G$.

Proof. Let $F(G, M)$ be the linear space of all $M$-valued functions on $G$ whose value is 0 outside a finite set of $G$. Define an $M_{*}$-sesquilinear form $[\cdot, \cdot]$ on $F(G, M)$ by

$$
[f, g]=\sum_{s} \sum_{t} f(s) \cdot V\left(t^{-1} s\right) \cdot g(t)^{*}
$$

and put $(f, g)=\langle 1,[f, g]\rangle$ for all $f, g$ in $F(G, M)$. Then $[\cdot, \cdot]$ is positive, and $(\cdot, \cdot)$ is a semidefinite inner product on $F(G, M)$. For $a$ in $M, t$ in $G$, define linear maps $\pi(a), U(t)$ on $F(G, M)$ by the relations

$$
\begin{aligned}
& (\pi(a) f)(s)=a f(s) \\
& (U(t) f)(s)=f\left(t^{-1} s\right)
\end{aligned}
$$

for all $f$ in $F(G, M), s$ in $G$. Then it is easy to see that

$$
\begin{aligned}
& {[\pi(a) f, g]=a \cdot[f, g]} \\
& {[U(t) f, U(t) g]=[f, g]}
\end{aligned}
$$

for all $f, g$ in $F(G, M)$. Then the subset $N=\{f$ in $F(G, M) ;[f, f]=0\}$ is invariant under $\pi(a)$ and $U(t)$. Let $W$ be the Hilbert space obtained by completing $F(G, M) / N$. Then by Lemma 3.6, $\pi$ induces a non-degenerate normal *-representation of $M$ on $W$, and similarly $U$ induces a unitary representation of $G$. Since it is easy to see that $\pi(a) U(t) f=U(t) \pi(a) f$ for all $f$ in $F(G, M)$, the condition (i) is clearly satisfied. Let $\xi$ be the vector in $W$ induced from $1_{e}$ in $F(G, M)$ such that $1_{e}(e)=1$ and that $1_{e}(s)=0$ if $s \neq e$, where $e$ is the unit of $G$. Then clearly the set $\left\{\pi(a) U(t) 1_{e} ; a\right.$ in $M, t$ in $\left.G\right\}$ spans $F(G, M)$ and hence the condition (ii) is obvious. Observing that $\left[U(t) 1_{e}, 1_{e}\right]=V(t)$, we have 
that $\langle a, V(t)\rangle=\left(\pi(a) U(t) 1_{e}, 1_{e}\right)$, and so we obtain the condition (iii). Now we have only to show the strong continuity of $U$. Since the routine calculus shows that

$$
(U(u) f, g)=\sum_{s} \sum_{t}\left\langle g(t)^{*} f(s), V\left(t^{-1} u s\right)\right\rangle
$$

for all $u$ in $G, f, g$ in $F(G, M)$, the weak continuity of $U$ implies the weak continuity of $U$. Thus the strong continuity of $U$ is concluded from the fact that the strong and weak topologies coincide on the unitary group of $W$.

Q.E.D.

The above construction of a unitary representation is a variation of that found by Umegaki [10].

\section{The structure of Hilbert $B(H)$-modules.}

In this section we shall study the structure of Hilbert $B(H)$-modules, and show that Hilbert $B(H)$-modules have a quite similar structure to that of usual Hilbert spaces except that $B(H)$ is not a field.

Let $\left\{X_{i}\right\}$ be a family of Hilbert $B(H)$-modules and let $\sum_{i} X_{\imath}$ be the Hilbert space direct sum of $\left\{X_{i}\right\}$ which is a left $B(H)$-module in the obvious way. Let $x=\left(x_{\imath}\right)$ and $y=\left(y_{\imath}\right)$ be in $\sum_{i} X_{\imath}$. By Proposition 3.4 we have $\left\|\left[x_{\imath}, y_{\imath}\right]\right\|_{1}$ $\leqq\left\|x_{i}\right\|_{2}\left\|y_{i}\right\|_{2}$, where $[\cdot, \cdot]$ is the Gramian on $X_{\imath},\|\cdot\|_{1}$ is the trace norm on $T(H)$, and $\|\cdot\|_{2}$ is the Hilbert space norm on $X_{2}$. Then it is easy to see that the family of trace class operators $\left\{\left[x_{\imath}, y_{\imath}\right]\right\}$ is summable in the trace norm. Now we define the Gramian on $\sum_{i} X_{\imath}$ by $[x, y]=\sum_{\imath}\left[x_{\imath}, y_{\imath}\right]$. Then we have that $(x, y)=\sum_{i}\left(x_{\imath}, y_{\imath}\right)=\operatorname{Tr}[x, y]$, and hence $\sum_{i} X_{\imath}$ is a Hilbert $B(H)$-module which is called the direct sum of the family $\left\{X_{i}\right\}$ of Hilbert $B(H)$-modules.

Lemma 4.1. Let $X$ and $Y$ be two Hilbert $B(H)$-modules, and $U$ be a map from $X$ onto $Y$. Then the following three conditions are equivalent:

(i) $U$ is an isomorphism from $X$ to $Y$;

(ii) $U$ is a unitary operator from $X$ to $Y$ such that $a \cdot U x=U(a \cdot x)$ for all $x$ in $X$;

(iii) $U$ satisfies $[U x, U y]=[x, y]$ for all $x, y$ in $X$.

Proof. It is trivial that (i) implies (ii). The routine calculus shows that (ii) implies that $\operatorname{Tr}(a[U x, U y])=\operatorname{Tr}(a[x, y])$ for all $a$ in $B(H), x, y$ in $H$. Thus (ii) implies (iii). It is easy to verify that (iii) implies that $[U(x+y)-U x$ $-U y, U(x+y)-U x-U y]=0$ and that $[U(a \cdot x)-a \cdot U x, U(a \cdot x)-a \cdot U x]=0$ for all $a$ in $B(H), x, y, z$ in $X$. Thus clearly (iii) implies (i).

Q.E. D.

THEOREM 4.2. Every Hilbert $B(H)$-module $X$ is equivalent to a direct sum $\Sigma H$ of (possibly infinitely many) copy of the Hilbert $B(H)$-modules $H$. 
Proof. By Lemma 3.6 there is a non-degenerate normal *-representation $\pi$ of $B(H)$ on the Hilbert space $X$ such that $(\pi(a) x, y)=\langle a,[x, y]\rangle$ for all $x, y$ in $X$. Since every *-representation of the $C^{*}$-algebra $C(H)$ of all compact operators on $H$ is unitarily equivalent to a direct sum of the identity representation on $H$, and since $C(H)$ is weakly dense in $B(H)$, we can conclude that $\pi$ is untarily equivalent to a direct sum of the identity representation of $B(H)$ on $H$. Thus by Lemma 4.1 this unitary equivalence induces the equivalence between two Hilbert $B(H)$-modules $X$ and $\Sigma H$.

Q.E.D.

COROLlary 4.3. Every Hilbert $B(H)$-module $X$ is equivalent to the Hilbert $B(H)$-module $S(K, H)$ for some Hilbert space $K$.

Proof. By Theorem 4.2 there is an index set $I$ such that $X$ is equivalent to $\sum_{\imath}\left\{H_{\imath} ; i\right.$ in $\left.I\right\} \quad\left(H_{\imath}=H\right.$ for all $\left.i\right)$. Let $K$ be a Hilbert space with basis $\left\{\xi_{\imath} ; i\right.$ in $\left.I\right\}$. For every element $x=\left(x_{\imath}\right)\left(x_{\imath}\right.$ in $\left.H\right)$, define an operator $U x=$ $\sum_{\imath} x_{i} \otimes \bar{\xi}_{\imath}$ from $K$ to $H$. Then it is easy to verify that $U x$ is in $S(K, H)$ and that the correspondence $U: x \rightarrow U x$ is an isomorphism from $X$ onto $S(K, H)$.

In order to proceed to the Fourier expansion of elements of a Hilbert $B(H)$-module, in which the Fourier coefficients are given by the Gramian, we shall define the basis of the Hilbert $B(H)$-module, as follows,

Definition 4.4. Let $\left\{x_{i}\right\}$ be a family of elements of a Hilbert $B(H)$ module $X$. We say that $\left\{x_{i}\right\}$ is modular othonormal if

(i) $\left[x_{\imath}, x_{3}\right]=0$, if $i \neq j$,

(ii) $\left[x_{\imath}, x_{\imath}\right]^{2}=\left[x_{\imath}, x_{\imath}\right]$ and $\left\|x_{i}\right\|_{2}=1$ for each $\imath$.

A maximal modular orthonormal family is called a modular basıs.

By Zorn's lemma, every Hilbert $B(H)$-module has a modular basis.

THEOREM 4.5. The following conditzons for a modular orthonormal family $\left\{x_{i}\right\}$ of elements of a Hilbert $B(H)$-module $X$ are all equivalent.

(i) The family $\left\{x_{i}\right\}$ is a modular basis of $X$.

(ii) If $x$ is in $X$ and if $\left[x, x_{2}\right]=0$ for all $i$, then $x=0$.

(iii) If, for each $i, X_{\imath}$ is the set $\left\{a \cdot x_{\imath} ; a\right.$ is in $\left.B(H)\right\}$, then $X=\sum_{\imath} X_{\imath}$ (the Hilbert space direct sum).

(iv) For all $x$ in $X, x=\sum_{i}\left[x, x_{\imath}\right] \cdot x_{\imath}$.

(v) For all $x$ in $X,[x, y]=\sum_{\imath}\left[x, x_{\imath}\right]\left[x_{\imath}, y\right]$, where the infinite sum is defined as unconditionaly covergence in $\|\cdot\|_{1}$-norm.

(vi) For all $x$ in $X,[x, x]=\sum_{i}\left|\left[x_{\imath}, x\right]\right|^{2}$, where $|\cdot|$ is such that $|a|=\left(a^{*} a\right)^{1 / 2}$. 
Proof. (i) implies (ii): If $x$ is in $X$, if $\left[x, x_{\imath}\right]=0$ for all $i$, and if $x \neq 0$, then the non-zero positive trace class operator $[x, x]$ on $H$ has a positive eigen value $\lambda$ with eigen vector $\phi,\|\phi\|_{2}=1$. Let $a$ be the operator on $H$ defined by $a \xi=\lambda^{-1 / 2}(\xi, \phi) \phi$ for all $\xi$ in $H$, and $x_{0}$ be such an element in $X$ that $x_{0}=a \cdot x$. Then the routine calculus shows that $\left[x_{0}, x_{0}\right]^{2}=\left[x_{0}, x_{0}\right]$ and that $\left\|x_{0}\right\|_{2}=1$. Thus we can add $x_{0}$ to the family $\left\{x_{i}\right\}$. This contradicts the assumed maximality of the family.

(ii) implies (iii): If $X \neq \sum_{\imath} X_{\imath}$, then there is a vector $x$ in $X$ such that $(x, y)=0$ for all $y$ in $\sum_{\imath} X_{\imath}$ but that $x \neq 0$. Thus

$$
\left\|\left[x, x_{\imath}\right]\right\|_{1}=\operatorname{Tr}\left(u\left[x, x_{\imath}\right]\right)=\left(x, u^{*} \cdot x_{\imath}\right)=0
$$

where $u$ is an partial isometry on $H$, and hence $\left[x, x_{\imath}\right]=0$ for all $\imath$, since $u^{*} x_{2}$ is in $X_{2}$. This contradicts (ii).

(iii) implies (iv): Assuming (iii), any $x$ in $X$ can be written as $x=\sum_{2} a_{\imath} \cdot x_{2}$ where $a_{\imath}$ is in $B(H)$. Then a routine calculus using the fact that $\left[x_{\imath}, x_{\imath}\right] \cdot x_{\imath}=x_{\imath}$ shows that $a_{1} \cdot x_{2}=\left[x, x_{2}\right] \cdot x_{2}$. Thus we have the required formula.

The remaining part of the proof is now an easy matter.

Q.E. D.

COROLlary 4.6 Any two modular basis of a Hilbert $B(H)$-module have the same power.

Proof. Let $\left\{x_{\imath} ; \imath\right.$ in $\left.I\right\},\left\{y_{\jmath} ; \jmath\right.$ in $\left.J\right\}$ be two modular basis of a Hilbert $B(H)$-module $X$. Then from (iii) of Theorem 4.5, we can see that $X$ is equivalent to $\sum_{\imath} H_{\imath}$ and $\sum_{j} H_{\jmath}$, where $H_{\imath}=H_{j}=H$, since $X_{\imath}=\left\{a \cdot x_{\imath} ; a\right.$ in $\left.B(H)\right\}$ (or $=\left\{a \cdot y_{\jmath} ; a\right.$ in $\left.B(H)\right\}$ ) is equivalent to $H$. Thus the powers of $I$ and $J$ are same, since they are two multiplicities of the two unitarily equivalent representations of $B(H)$.

Q.E. D.

The common power of all modular basis of a Hilbert $B(H)$-module $X$ is called the modular dimention of $X$ and written as $\operatorname{Dim}(X)$.

The following Theorem is now an immediate consequence of Theorem 4.2.

THEOREM 4.7. Two Hilbert $B(H)$-modules are equivalent if and only if they have the same modular dimension.

It should be remarked that $\operatorname{Dim}(X) \cdot \operatorname{dim}(H)=\operatorname{dim}(X)$ and that $\operatorname{Dim}(S(K, H))$ $=\operatorname{dim}(K)$, where $\operatorname{dim}(\cdot)$ is the usual dimention of Hilbert spaces.

\section{Equivalence of $G$-stationary processes.}

In the following, we shall consider a fixed locally compact group $G$. Recall that a $T(H)$-valued function $V$ on $G$ is positive definite if and only if for any positive integer $n$, we have that $\sum_{\imath, j} \operatorname{Tr}\left(a_{j}^{*} a_{\imath} V\left(t_{j}{ }^{-1} t_{\imath}\right)\right) \geqq 0$ for all 
$a_{1}, \cdots, a_{n}$ in $B(H), t_{1}, \cdots, t_{n}$ in $G$. Let $X$ be a Hilbert $B(H)$-module, and let $\left\{x_{t}\right\}$ be a $G$-stationary process on $X$. Then it is easy to see that the covariance function $\Gamma$ of the process $\left\{x_{t}\right\}$ is a $T(H)$-valued positive definite function on $G$. Now we shall show that every positive definite $T(H)$-valued function is the covariance function of some, but unique up to equivalence, $G$-stationary process on a Hilbert $B(H)$-module.

THeOREm 5.1. Let $\Gamma$ be a weakly contrnuous positive definte $T(H)$-valued function on $G$. Then there exist a Hilbert $B(H)$-module $X$ and a G-stationary process $\left\{x_{t}\right\}$ on $X$ whose covariance function is $\Gamma$. In this case there is a strongly continuous unitary representation $U$ of $G$ on $X$ such that $x_{t}=U(t) x_{e}$ and that $U(t)(a \cdot x)=a \cdot U(t) x$ for all $t$ on $G, a$ in $B(H), x$ in $X$.

Proof. Applying Theorem 3.7 to the case $M=B(H)$, we have a Hilbert $B(H)$-module $X$, a non-degenerate normal *-representation $\pi$ of $B(H)$ on $X$, a strongly continuous unitary representation $U$ of $G$ on $X$ and a vector $x_{e}$ in $X$ which satisfy the conditions in that theorem. Put the process $\left\{x_{t}\right\}$ as $x_{t}=$ $U(t) x_{e}$ for all $t$ in $G$, and define the Gramian $[\cdot, \cdot]$ on $X$ by $\operatorname{Tr}(a[x, y])$ $=(\pi(a) x, y)$ for all $a$ in $B(H), x, y$ in $X$. Then the routine calculus shows that

$$
\operatorname{Tr}\left(a \Gamma\left(t^{-1} s\right)\right)=\left(\pi(a) U(s) x_{e}, U(t) x_{e}\right)=\operatorname{Tr}\left(a\left[x_{s}, x_{t}\right]\right)
$$

for all $a$ in $B(H), s, t$ in $G$. Thus the conclusion follows from Theorem 3.7.

Q.E. D.

Theorem 5.2. Let $X$ and $Y$ be two Hilbert $B(H)$-modules. Let $\left\{x_{t}\right\}$ be a $G$-stationary process on $X$ with covariance function $\Gamma_{x}$, and let $\left\{y_{t}\right\}$ be a Gstationary process on $Y$ with convariance function $\Gamma_{y}$. Then $\left\{x_{t}\right\}$ and $\left\{y_{t}\right\}$ are equivalent if and only if $\Gamma_{x}(t)=\Gamma_{y}(t)$ for all $t$ in $G$.

Proof. Since the "only if" part is trivial, we assume $\Gamma_{x}(t)=\Gamma_{y}(t)$ for all $t$ in $G$. First we observe that

$$
\begin{aligned}
{\left[\sum_{\imath} a_{\imath} \cdot x_{t_{i}}, \sum_{\jmath} b_{\jmath} \cdot x_{s_{\jmath}}\right] } & =\sum_{\imath, \jmath} a_{i} \Gamma_{x}\left(s_{\jmath}{ }^{-1} t_{\imath}\right) b_{\jmath}{ }^{*} \\
& =\left[\sum_{\imath} a_{\imath} \cdot y_{t_{i}}, \sum_{\jmath} b_{\jmath} \cdot y_{s_{\jmath}}\right]
\end{aligned}
$$

for all $a_{\imath}, \cdots, a_{n}, b_{1}, \cdots, b_{m}$ in $B(H), t_{1}, \cdots, t_{n}, s_{1}, \cdots, s_{m}$ in $G$, and given $n, m$. Thus putting $U^{\prime}\left(\sum_{\imath} a_{\imath} \cdot x_{t_{\imath}}\right)=\sum_{\imath} a_{\imath} \cdot y_{t_{i}}$, we can define a map $U^{\prime}$ from $X^{\prime}$ onto $Y^{\prime}$ such that $\left[U^{\prime} x, U^{\prime} y\right]=[x, y]$ for all $x, y$ in $X^{\prime}$, where $X^{\prime}$ and $Y^{\prime}$ are submodules spanned by $\left\{x_{t}\right\}$ and $\left\{y_{t}\right\}$, respectively. Then since $U^{\prime}$ is isometry, and since $X^{\prime}$ and $Y^{\prime}$ are dense in $X$ and $Y$, we can extend $U^{\prime}$ to a map $U$ on $X$ onto $Y$ such that $[U x, U y]=[x, y]$ for all $x, y$ in $X$. Thus the conclusion follows from Lemma 4.1 .

Q.E. D. 
From Theorem 4.3, every $G$-stationary process on a Hilbert $B(H)$-module $X$ can be regarded as a $G$-stationary process on the Hilbert $B(H)$-module $S(K, H)$ for some Hilbert space $K$ with $\operatorname{dim}(K)=\operatorname{dim}(X)$. Thus the following theorem shows that every $G$-stationary process on a Hilbert $B(H)$-module $X$ is equivalent to such as given by the theorem.

THEOREM. 5.3. Let $K$ be a Hilbert space, and let $\left\{x_{t}\right\}$ be a G-stationary process on the Hilbert $B(H)$-module $S(K, H)$ with covariance function $\Gamma$. Then there is a strongly continuous unitary representation $U$ of $G$ on $K$ such that $x_{t}=x_{e} U(t)^{*}$ for all $t$ in $G$, where $x_{e} U(t)^{*}$ is the product of two operators $x_{e}$ and $U(t)^{*}$, and that $\Gamma(t)=x_{e} U(t)^{*} x_{e}^{*}$.

Proof. Let $H \otimes K$ be the tensor product Hilbert space of $H$ and $K$, and fix a basis $\left\{\phi_{i}\right\}$ of $K$. Then we can identify $S(K, H)$ and $H \otimes K$ by the correspondence $\sum_{\imath} \phi_{i} \otimes \bar{\psi}_{i} \rightarrow \sum_{\imath} \phi_{i} \otimes \phi_{i}$. By Theorem 5.1 and 5.2, we have a strongly continuous unitary representation $V$ of $G$ on $H \otimes K$ such that $x_{t}=V(t) x_{e}$ and that $V(t) a \cdot x=a \cdot V(t) x$ for all $t$ in $G, x$ in $S(K, H), a$ in $B(H)$. Since the all actions of $B(H)$ on $H \otimes K$ constitutes the von Neumann algebra $B(H) \otimes 1$ on $H \otimes K$, and $V(G)$ is contained in $(B(H) \otimes 1)^{\prime}$ which is equal to $1 \otimes B(K)$, we have a strongly continuous unitary representation $U$ of $G$ on $K$ such that $V(t)=1 \otimes U(t)$ for all $t$ in $G$. Thus the conclusion follows from the computations that

$$
\begin{aligned}
x_{t}=V(t) x_{e} & =(1 \otimes U(t)) \sum_{\imath} \phi_{i} \otimes \bar{\psi}_{i}=\sum_{\imath} \phi_{i} \otimes \overline{U(t) \phi_{i}} \\
& =\left(\sum_{\imath} \phi_{i} \otimes \overline{\phi_{i}}\right) U(t)^{*}=x_{e} U(t)^{*}
\end{aligned}
$$

where $\phi_{i}=x_{e} \phi_{\imath}$ for all $i$, and that

$$
\Gamma(t)=\left[x_{t}, x_{e}\right]=x_{t} x_{e}^{*}=x_{e} U(t)^{*} x_{e}^{*} . \quad \text { Q. E. D. }
$$

\section{REFERENCES}

[1] CHOI, M.D., A Schwartz inequality for positive linear maps on $C^{*}$-algebras, Illinois J. Math. 18 (1974), 565-574.

[2] Halmos, P.R., Introduction to Hilbert spaces and the theory of spectral multiplicity, Chelsea, New York, 1951.

[3] Kallianpur, G. And Mandrekar, V., Spectral theory of stationary $H$-valued processes, J. Multivariate Anal. 1 (1971), 1-16.

[4] Kolmogorov, A. N., Stationary sequences in Hilbert spaces, Bull. Math. Univ. Moscow, 2 (1941), 1-40.

[5] MASANI, P., Recent trends in multivariate prediction theory, in Krishnaiah, P. R., Multivariate Analysis, Academic Press, New York, 1966.

[6] PAschke, W. L., Inner product modules over $B^{*}$-algebras, Trans. Amer. Math. Soc. 182 (1973), 443-468. 
[7] Payen, R., Fonctions aleatoires du second ordre a valeurs dans un espace de Hilbert, Ann. Inst. H. Poincare 3 (1967), 323-396.

[8] SAKAI, S., $C^{*}$-algebras and $W^{*}$-algebras, Ergebn. Math. Grenzgeb. Vol. 60 (Springer, Berlin, 1971).

[9] Schatten, R., Norm ideals of completely continuous operators, Springer, Berlin, 1960.

[10] Umegaki, H., Positive definite function and direct product Hilbert space, Tohoku Math. J. 7 (1955), 206-211.

[11] UMEgAKI, H. AND BHARUCHA-REID, A.T., Banach space-valued random variables and tensor products of Banach spaces, J. Math. Anal. Appl. 31 (1970), 49-67.

[12] Stinespring, W.F., Positive functions on $C^{*}$-algebras, Proc. Amer. Math. Soc. 6 (1955), 211-216.

DEPARTMENT OF INFORMATION SCIENCES

Tokyo Institute of Technology

OH-OKayama, Meguro-Ku, TOKYo, Japan 